\title{
MODELO TRIPARTITO SOBRE EL AFECTO POSITIVO Y NEGATIVO, LA DEPRESIÓN Y LA ANSIEDAD: EVIDENCIA BASADA EN LA ESTRUCTURA DE LOS SÍNTOMAS Y EN DIFERENCIAS SEXUALES
}

\author{
Thomas E. Joiner, Jr.', Salvatore J. Catanzaro², Jeff Laurent ${ }^{2}$, \\ Bonifacio Sandin ${ }^{3}$ y Janice A. Blalock ${ }^{1}$ \\ University of Texas Medical Branch at Galveston, Galveston, Texas, USA \\ 2Illinois State University, Normal, Illinois, USA \\ ${ }^{3}$ Universidad Nacional de Educación a Distancia, Madrid, España
}

\begin{abstract}
RESUMEN
El modelo tripartito sobre la depresión y la ansiedad sugiere que la depresión y la ansiedad poseen componentes compartidos (afecto negativo generalizado) y especificos (anhedonia e hiperactivación fisiológica). En este artículo comentamos cinco estudios recientes que han abordado aspectos del modelo tripartito. Los estudios indican que: 1) el afecto positivo y el afecto negativo representan dimensiones separables del ánimo, lo cual es evidenciable a través de diferentes culturas incluida la española; 2) el modelo tripartito de la depresión y la ansiedad parece ser una buena descripción de las características de ambos sindromes; 3 ) hombres y mujeres parecen diferir respecto a los sindromes asociados a la depresión, pero no en relación con la depresión "pura" en si misma. Se discuten la implicaciones teóricas y clínicas de estos hallazgos.
\end{abstract}

PALABRAS CLAVE: depresión, ansiedad, modelo trtipartito, afecto positivo, afecto negativo, PANAS

\section{ABSTRACT}

The tripartite model of depression and anxiety suggests that depression and anxiety possess shared (generalized negative affect) and specific (anhedonia and physiological hyperarousal) components. In this paper, we describe five recent studies that have addressed aspects of the tripartite model. The studies indicate that: 1) positive affect and negative affect represent separable dimensions of mood across cultures, including Spain; 2) the tripartite model of depression and anxiety appears to be a valid description of the features of the two syndromes; 3 ) women and men may differ regarding depressionrelated syndromes, but not regarding "pure" depression itself. Theoretical and clinical implications are discussed. PANAS

KEY WORDS: depression, anxiety, tripartite model, positive affect, negative affect,

Correspondencia: Dr. Thomas Joiner, D-25, Department of Psychiatry and Behavioral Science, Graves Building, University of Texas. Medical Branch at Galveston, Galveston, Texas, 77555-0425, USA. Fax. 409-747-1311. E-mail: Thomas Joiner @UTMB.edu. 


\section{INTRODUCCIÓN}

Los trastornos de ansiedad y depresivos frecuentemente ocurren de forma simultánea, es decir, son trastornos comórbidos. Las personas depresivas y ansiosas con frecuencia se comportan y exhiben características similares. Así, ambos tipos de individuos pueden ser descritos como disgustados, tensos, estresados, preocupados, agitados, con dificultades para dormir, etc. Pero, ¿qué es lo que distingue la depresión de la ansiedad?

Para abordar esta cuestión es preciso conocer la estructura del afecto. Este problema posee una larga historia. Sirva de ejemplo cómo en el siglo XIII los teólogos escolásticos, incluido el propio Santo Tomás de Aquino, intentaron llevar a cabo una categorización de las pasiones básicas. Su esquema clasificatorio se reducía a dos categorias superiores tristitia y gaudium (tristeza y alegría). Es de interés observar que tristitia y gaudium eran reconocidas como polos opuestos, de tal forma que la presencia de una implicaba la ausencia de la otra (véase Jackson, 1985, pp. 49-50).

Es interesante resaltar que Platón abordó también este problema en la República, si bien llega a una conclusión diferente a la de Santo Tomás de Aquino. El principal objetivo de Platón era describir una sociedad ideal, en la cual el gobierno residiese en manos de filósofos-reyes. A modo de dilucidación de las características de los reyes-filósofos, Platón, hablando sobre el carácter de Sócrates, argumentaba que los placeres de los filósofos-reyes eran puros, descontaminados del dolor y de los placeres básicos. Puesto que los placeres elevados eran separables de los otros afectos, Platón concluye que no puede ser "correcto suponer que la ausencia de dolor sea placer o ausencia de dolor satisfactorio" (584-A). En otras palabras, Platón se decanta por un modelo bidimensional mejor que por un modelo unidimensional del afecto.

El trabajo de Watson, Clark, Tellegen y colaboradores sobre la estructura del afecto (e.g., Watson, Clark y Tellegen, 1988) se aproxima al punto de vista bidimensional de Platón; sin embargo, también se han señalado algunos aspectos críticos a esta orientación (e.g., Green, Goldman y Salovey, 1993). Watson et al. han referido datos que sugieren que la afectividad positiva y negativa no son caras de la misma moneda, sino que están separadas y pueden existir y funcionar de modo independiente. Este resultado -que la afectividad positiva y la negativa no están entrelazadas- ha sido suficientemente validado (e.g.; Watson, 1988), y replicado transculturalmente (e.g., Watson, Clark y Tellegen, 1984, con muestras japonesas).

Tales resultados poseen importantes implicaciones con respecto a los trastornos de ansiedad y del estado de ánimo. Clark y Watson (1991) y Watson, Clark y Carey (1988) han sugerido que la depresión "pura" se caracteriza por presentar baja afectividad positiva (i.e., anhedonia) y alta afectividad negativa. En contraste, la ansiedad "pura" se caracterizaba por la presencia de elevada hiperactivación fisiológica y alta afectividad negativa. Por consiguiente, la depresión y la ansiedad "puras" poseen un componente común (el afecto negativo), y componentes especificos (bajo afecto positivo y alta hiperactivación fisiológica, respectivamente). Este modelo ha sido denominado "modelo tripartito de la depresión y la ansiedad".

Los componentes del modelo tripartito pueden ser utilizados para entender la combinación entre depresión y ansiedad, así como también la distinción entre ambas. Por ejemplo, el trastorno mixto de ansiedad-depresión, que ha sido propuesto por el DSM-IV (American Psychiatric Association, 1994) como una categoria diagnóstica que requiere futuras consideraciones, se caracteriza por presentar un incremento del componente compartido (no especifico) del afecto negativo, y una ausencia relativa en cuanto al incremento de los componentes especificos de anhedonia e hiperactivación fisiológica (para mayor información sobre el síndrome mixto de ansiedaddepresión, véase Zinbarg et al., 1994).

La finalidad del presente artículo consiste en revisar cinco de nuestros recientes estudios vinculados de forma relevante al modelo tripartito sobre la depresión y la ansiedad. EI primer estudio (Joiner, Sandín, Chorot, Lostao y Marquina, 1995) refiere resultados sobre una medida del afecto positivo y negativo basados en una muestra extensa de mujeres en España. Los estudios segundo y tercero (Joiner, en prensa; Joiner, Catanzaro y Laurent, en pren- 
sa) informan sobre datos relacionados con la estructura tripartita de los sintomas asociados al estado de ánimo. Los estudios cuarto y quinto (Joiner y Blalock, en prensa; Joiner, Blalock y Wagner, 1995) se focalizan en las diferencias sexuales respecto a los tres componentes del modelo tripartito.

\section{AFECTO POSITIVO Y NEGATIVO EN UNA MUESTRA DE MUJERES ESPAÑOLAS}

La conclusión de que el afecto positivo y el negativo comprenden una estructura bidimensional del afecto ha sido apoyada transculturaimente. Usando la técnica $P$ de análisis factorial (i.e., análisis intrasujetos a través del tiempo) con los datos de una muestra japonesa, Watson et al. (1984) constataron la presencia de una clara estructura bifactorial, cuyos dos factores representaban al afecto positivo y al afecto negativo, respectivamente. Esta estructura del afecto obtenida a partir de la muestra japonesa era semejante a la encontrada con muestras norteamericanas (USA).

Si bien su trabajo no estaba específicamente orientado a estudiar la estructura del modelo del afecto positivo versus negativo, Russell (1983) también encontró que se obtenía un modelo bifactorial del afecto, incluso con datos obtenidos a partir de cinco idiomas diferentes (i.e., chino, croata, inglés, guajarati y japonés). El modelo del afecto positivo afecto negativo también ha sido apoyado empiricamente sobre la base de muestras francesas (D. Moskowitz, comunicación personal, 9 de marzo de 1995), israelies (Almagor y Ben-Porath, 1989) y rusas (Batalsky y Diener, 1993; Leon, Kanfer, Hoffman y Dupre, 1991). Es sorprendente, sin embargo, que no se hayan publicado datos basados en muestras de habla española'.

El objetivo del estudio de Joiner, Sandín, Chorot, Lostao y Marquina (1995) consistió en llevar a cabo una validación preliminar del
SPANAS (Spanish Positive and Negative Affect Schedule), versión española del questionario de Watson, Clark y Tellegen (1988) sobre el afecto positivo y negativo. En el proceso del desarrollo de esta medida, nosotros esperábamos validar la estructura bifactorial del afecto positivo y negativo partiendo de un nuevo grupo cultural, esto es, a partir de una muestra de mujeres españolas.

LLevando a cabo técnicas de análisis factorial confirmatorio sobre datos obtenidos con una muestra de 708 mujeres del norte de España, obtuvimos evidencia demostrativa de la existencia de validez de constructo y de fiabilidad razonables para esta nueva medida, la SPANAS. Consistente con previos estudios transculturales, de los datos de esta investigación se obtuvo una estructura claramente bifactorial de afecto positivo - afecto negativo, apareciendo el afecto positivo y el afecto negativo como dos entidades relativamente independientes. Se evidencia, por tanto, que esta estructura factorial se mantiene invariante a través de múltiples grupos culturales en Asia, Europa y Norte América.

ESTRUCTURA TRIPARTITA DE LOS SINTOMAS ASOCIADOS AL ESTADO DE ÁNIMO EN ESTUDIANTES UNIVERSITARIOS $Y$ PACIENTES PSIQUIÁTRICOS JÓVENES INTERNADOS

La investigación sobre el modelo tripartito basada en el análisis factorial ofrece cierta base como para suponer que una estructura trifactorial puede ser válida, al menos para población adulta. Por ejemplo, en el análisis de un cuestionario sobre sintomas del estado de ánimo y de ansiedad, Watson et al. (1995) obtuvieron tres factores que correspondian a las distinciones tripartitas de diestrés generalizado, depresión específica (anhedonia) y ansiedad especifica (hiperactivación fisiológica). Esta solución factorial, obtenida utilizando análisis factorial de componentes principales, se repitió consistentemente a través de pacien-

\footnotetext{
${ }^{1}$ Es preciso señalar, no obstante, que Sánchez-Cánovas (1994; Sánchez-Cánovas y Sánchez, 1994a, 1984b) ha construido un cuestionario sobre el afecto positivo y negativo basado, en parte, en los items originales procedentes del grupo de A. Tellegen y D. Watson. Este autor ha referido datos que apoyan la validez del modelo bidimensional del afecto.
} 
tes, no pacientes y de estudiantes universitarios (véase también Clark, Steer y Beck, 1994; Jolly y Dykman, 1994).
El propósito de los estudios de Joiner (en prensa) y Joiner, Catanzaro y Laurent (en prensa) consistió en investigar la estructura de

Figura 1. Resultados de análisis factorial confirmatorio LISREL del modelo tripartito obtenidos con una muestra de estudiantes universitarios. Las vias que conectan los óvalos representan interrelaciones de variables latentes; las vias desde los óvalos hasta los cuadros representan relaciones de las variables latentes sobre las observadas; las vías debajo de los cuadros representan los residuos error. $\mathrm{BDI}=$ Beck Depression Inventory. BAI = Beck Anxiety Inventory.

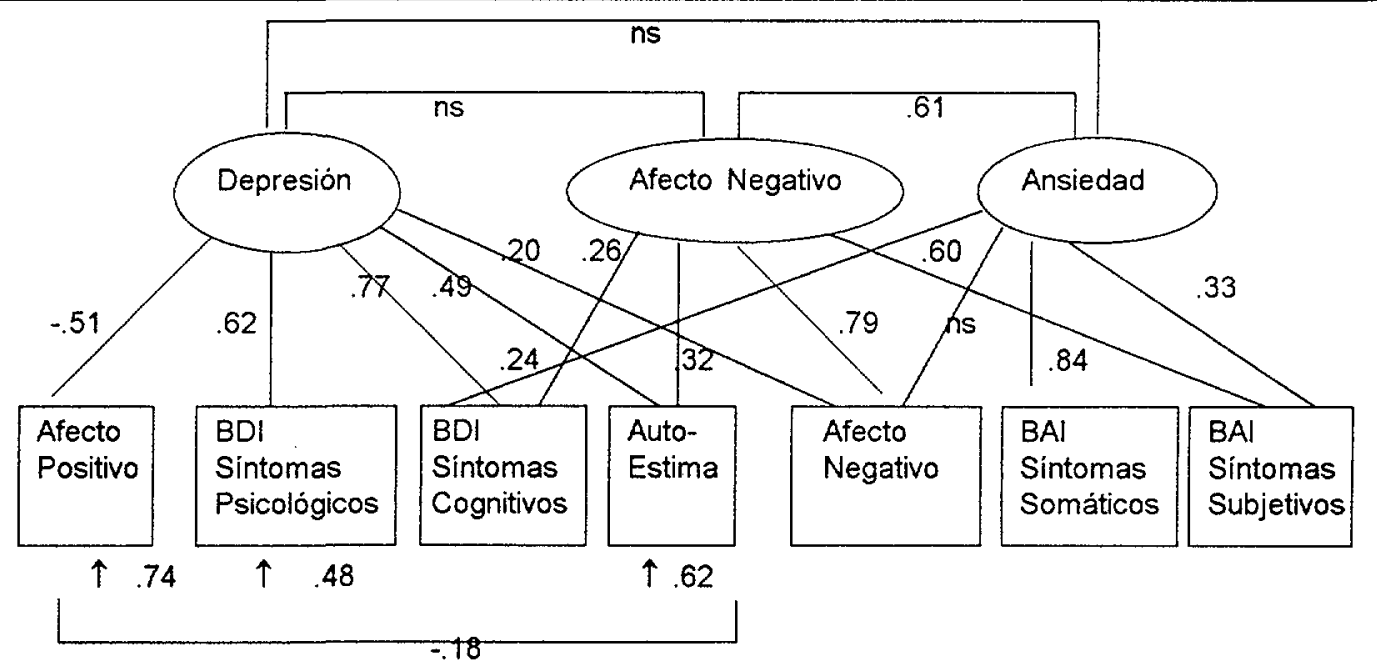

los síntomas asociados al estado de ánimo en muestras de estudiantes universitarios y muestras de pacientes psiquiátricos jóvenes (niños y adolescentes) respectivamente. En estos estudios también se pretendia determinar si la versión trifactorial del modelo tripartito de la depresión y la ansiedad podía ser apoyada empiricamente, en comparación con otros modelos alternativos.

Los resultados de ambos estudios, en general, apoyaban con bastante firmeza el modelo tripartito. Como puede apreciarse en la Figura 1 , un análisis factorial confirmatorio LISREL sobre datos de 205 estudiantes universitarios apoyan la existencia de un modelo trifactorial. El modelo trifactorial era superior a otros modelos como el unifactorial o el bifactorial. En el modelo trifactorial, el bajo afecto positivo era un indicador específico de la depresión; la hiperactivación fisiológica fue un indicador específico de la ansiedad; y los indicadores no específicos, tal como el ánimo negativo, configuraron el factor de afecto negativo.
Unas conclusiones semejantes fueron obtenidas a partir de la muestra de 116 pacientes psiquiátricos jóvenes, tal y como se indica en la Figura 2. Aqui, de nuevo, la estructura trifactorial resultó ser más apropiada que la basada en modelos uni- o bifactoriales.

Por tanto, parece deducirse que el modelo tripartito sobre la depresión y la ansiedad sugerido por Clark, Watson y colaboradores (e.g., Clark y Watson, 1991) resulta ser una descripción válida sobre los componentes particulares de los sindromes de la depresión y la ansiedad (i.e., anhedonia e hiperactivación fisiológica, respectivamente), así como también del componente que comparten ambos síndromes (i.e., afecto negativo general).

\section{DIFERENCIAS SEXUALES ASOCIADAS A LOS COMPONENTES DEL MODELO TRIPARTITO}

La incidencia de la depresión clínica y de los síntomas depresivos es entre dos y tres 
Figura 2. Modelo tripartito de la depresión y la ansiedad a partir de una muestra de internados psiquiátricos jóvenes, con estimaciones paramétricas de análisis factoriales confirmatorios LISREL. AP= Afecto Positivo. CDI= Children's Depression Inventory. RCMAS= Revised Children's Manifest Anxiety Scale.

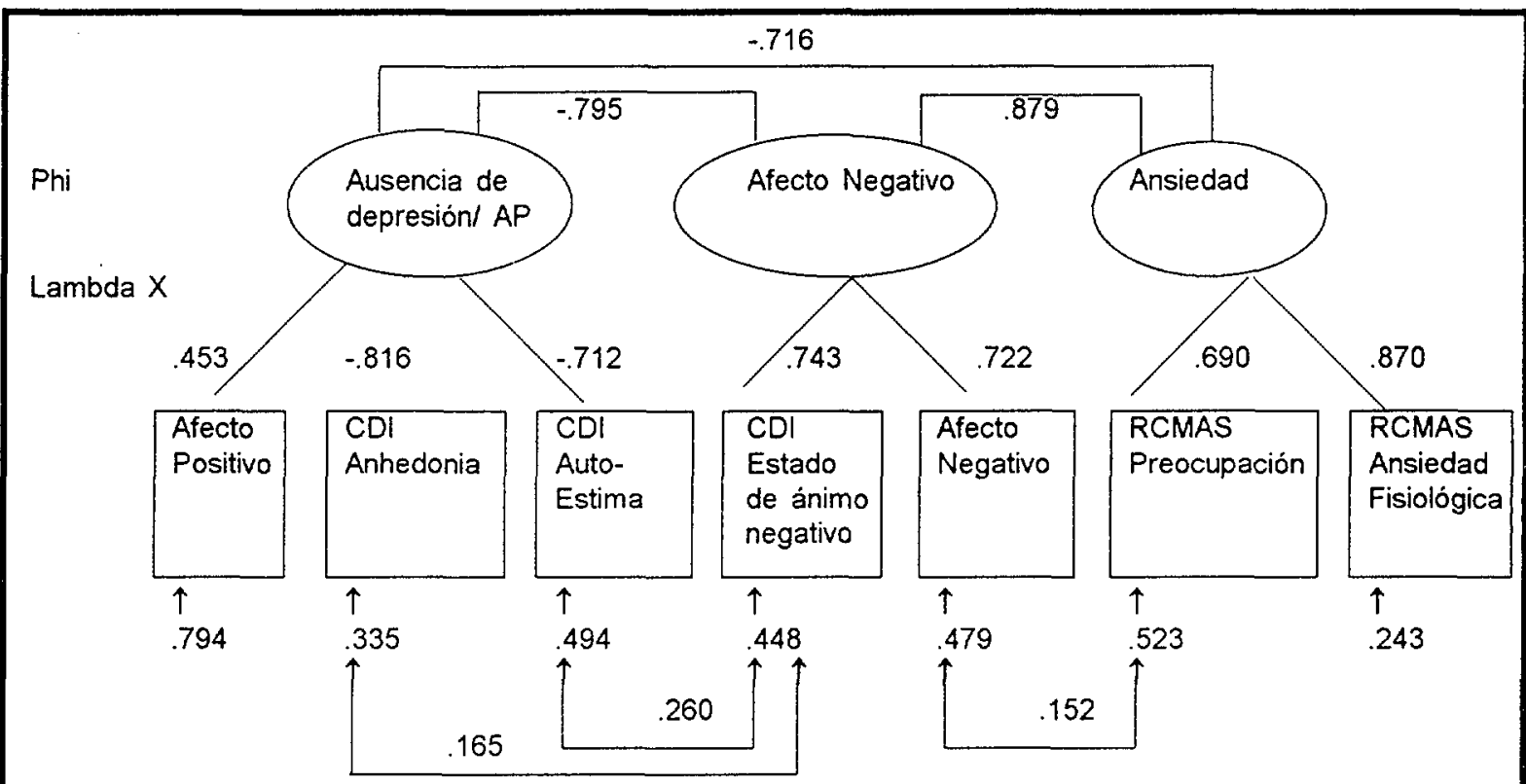

Theta Delta

veces superior en la mujer que en el hombre (Klerman y Weissman, 1989). Este fenómeno parece ser más o menos invariable: tiende a persistir incluso cuando variables demográficas como la raza, el nivel económico, la educación y la ocupación son controladas (McGrath, Keita, Strickland y Russo, 1991).

Se han ofrecido numerosas explicaciones alternativas para dar cuenta sobre las diferencias sexuales asociadas a la depresión, pero ninguna parece explicar totalmente la cuestión. El que múltiples orientaciones teóricas hayan sido incapaces de explicar conclusivamente las diferencias sexuales en la depresión sugiere que podría ser importante determinar en primer término si estas diferencias son específicas de la depresión propiamente dicha, o si, por el contrario, si ellas se relacionan con diversos sintomas, trastornos y rasgos con los que la depresión y el sexo covarian.

El objetivo de los estudios de Joiner y Blalock (en prensa) y Joiner, Blalock y Wagner (1995) consistió en analizar las diferencias sexuales con relación al modelo tripartito, partiendo de la hipótesis central de que todos los componentes excepto la depresión "pura" deberian ser más comunes entre las mujeres. Existen algunas líneas de evidencia que apoyan este punto de vista. Por ejemplo, Ochoa, Beck y Steer (1992) informaron que no existian diferencias sexuales relativas al diagnóstico de los trastornos del estado de ánimo, en aquellos individuos que no recibian ningún diagnóstico comórbido de algún trastorno de ansiedad. En contraste, existía una proporción mujer/varón de 2:1 para aquellas personas que recibian diagnósticos comórbidos de trastornos del estado de ánimo y de ansiedad. Este resultado sugiere que existen diferencias sexuales cuando existe comorbilidad entre ansiedad y depresión, pero no cuando se trata de depresión "específica" o "pura".

Nuestros resultados pertenecientes tanto al grupo de estudiantes universitarios como al de jóvenes pacientes psiquiátricos apoyan el punto de vista de que no existen diferencias sexuales con respecto a la depresión "pura", pero que las diferencias sexuales son sustanciales con 
respecto a los estados combinados de ansiedad, depresión y alto afecto negativo. Más específicamente, dentro de la muestra de estudiantes universitarios, la proporción de mujeres versus varones fue significativamente elevada en las categorias de depresión y ansiedad comórbidas y en el cuadro mixto de ansiedad-depresión, pero no en las categorias de depresión" especifica" ("pura"). Resultados similares fueron obtenidos en pacientes psiquiátricos internados (niños y adolescentes internados). Por tanto, parece concluirse que, mientras la depresión "específica" posee prevalencias semejantes en el hombre y en la mujer, otros sindromes relacionados con la depresión parecen ser más comunes entre las mujeres que en los varones.

\section{SUMARIO Y CONCLUSIONES}

Los cinco estudios comentados atrás indican que: 1) el afecto positivo y el afecto negativo constituyen dimensiones separables del estado de ánimo, lo cual ha sido observado a través de distintas culturas, incluida la española; 2) el modelo tripartito sobre la depresión y la ansiedad parece ser una descripción válida sobre las caracteristicas de ambos síndromes; 3) las mujeres y los varones pueden diferir en relación con los síndromes asociados a la depresión, pero no parece que sea asi respecto a la depresión "pura" como tal.

En cuanto a las implicaciones de estos estudios, el diagnóstico diferencial entre la depresión y la ansiedad a veces puede resultar dificil de establecer, especialmente entre personas durante edades infantiles, puesto que este tipo de pacientes en ocasiones son incapaces de describir en qué consiste específicamente su malestar subjetivo (diestrés), y porque ambos trastornos con frecuencia son fenomenológicamente similares entre sí. El modelo tripartito, asi como las herramientas psicométricas que lo asisten, aportan una estrategia útil para llevar a cabo una distinción, al menos con respecto a la depresión y la ansiedad.

Desde el punto de vista clínico, el modelo tripartito puede ser de utilidad para establecer con los pacientes estrategias de educación emocional (véase, por ejemplo, Kendall, Kortlander, Chansky y Brady, 1992). En consonancia con los enfoques cognitivos sobre el tratamiento de la depresión y la ansiedad, las personas que reconocen las diferencias entre depresión, ansiedad y afecto negativo generalizado pueden identificar y alterar de forma más eficaz las cogniciones que preceden a dichos estados emocionales. Así mismo, como han sugerido Kendall et al. (1992), existe cierta evidencia de que la ansiedad podria ser un precursor de la depresión (cf., Alloy, Kelly, Mineka y Clements, 1990; Dobson, 1985). Por tanto, la identificación previa de ansiedad "específica" podría servir como un indicador para llevar a cabo acciones que permitan evitar el comienzo de la depresión "específica".

El modelo tripartito también puede resultar útil para que los clínicos puedan entender mejor las bases de los síntomas y conductas asociadas al estado de ánimo, especialmente aquéllos comportamientos que implican déficits (e.g., déficits en habilidades sociales). Debido a que los pacientes a veces experimentan dificultades para transmitir y describir su malestar (diestrés) subjetivo, con frecuencia resulta difícil discernir la causa de un determinado déficit. Por ejemplo, la reticencia social evidenciada en una determinada persona puede deberse a una (depresotípica) falta de motivación, o a un (ansiotípico) miedo a la interacción social. En casos de este tipo, el modelo tripartito puede servir como árbitro (para una discusión detallada sobre cuestiones de tratamiento, véase Kendall et al., 1992; Stark, Rouse y Livingston, 1991).

¿Cuáles son las razones por las que la ansiedad y la depresión comórbidas yel cuadro mixto de ansiedad-depresión serían más comunes entre las mujeres que entre los varones? Aunque nuestros estudios no han abordado específicamente esta cuestión, podrian ofrecerse algunas consideraciones a este respecto. En primer lugar, existe evidencia de que las mujeres puntúan más elevado que los varones en el rasgo de emocionalidad (Buss, 1986), y de que la emocionalidad está influenciada tanto por factores genéticos como de socialización (Buss y Plomin, 1984). Resulta interesante señalar que el cuadro mixto ansioso-depresivo, asi como también la ansiedad y 
depresión comórbidas, requieren la existencia de hiperactividad emocional (negativa), mientras que la depresión "específica" implica hipoactividad emocional (i.e., anhedonia). Las mujeres, que posean mayor grado de emocionalidad, pueden también desarrollar con mayor probabilidad sindromes que incluyan hiperactividad de emociones negativas, pero no síndromes que incluyan hipoactividad para emociones positivas.

Un segundo tipo de especulación implicaría la certeza con que se efectúan atribuciones negativas sobre acontecimientos negativos. Alloy et al. (1990) hipotetizaron que los síntomas de ansiedad y depresión ocurrirán conjuntamente cuando un individuo lleva a cabo atribuciones negativas inciertas (inseguras) sobre eventos negativos, mientras que los sintomas depresivos aparecerán solos cuando la persona efectúa atribuciones negativas seguras sobre sucesos negativos. Es posible que la mujer sea menos decisiva que el hombre a la hora de establecer sus atribuciones negativas. Apoyo indirecto a este punto de vista deriva de algunas investigaciones que sugieren que la mujer posee mayor probabilidad que el hombre de llevar a cabo rumiaciones sobre acontecimientos y estados de ánimo negativos (e.g., Nolen-Hoeksema, Morrow y Fredrikson, 1993).

\section{REFERENCIAS BIBLIOGRÁFICAS}

Almagor, M., y Ben-Porath, Y. S. (1989). The twofactor model of self-reported mood: A crosscultural replication. Journal of Personality Assessment, 53, 10-21.

Alloy, L. B., Kelly, K. A., Mineka, S., y Clements, C. (1990). Comorbidity in anxiety and depressive disorders: A helplessness/hopelessness perspective. En J. D. Maser y C. R. Cloninger (Eds.), Comorbidity in anxiety and mood disorders. Washington, D.C.: American Psychiatric Press, Inc.

American Psychiatric Association (1994). Diagnostic and Statistical Manual of Mental Disorders (4th ed.). Washington, D.C.: Author.

Balatsky, G., y Diener, E. (1993). Subjective wellbeing among Russian students. Social Indicators Research, 28, 225-243.
Buss, A. H. (1986). Social behavior and personality. Hillsdale, NJ: Erlbaum.

Buss, A. H., y Plomin, R. (1984). Temperament: Early developing personality traits. Hillsdale, $\mathrm{NJ}$ : Erlbaum.

Clark, D. A., Steer, R. A., y Beck, A. T. (1994). Common and specific dimensions of self-reported anxiety and depression: Implications for the cognitive and tripartite models. Journal of Abnormal Psychology, 103, 645-654.

Clark, L. A., y Watson, D. (1991). Tripartite model of anxiety and depression:Psychometric evidence and taxonomic implications. Joumal of Abnormal Psychology, 100, 316-336.

Dobson, K. S. (1985). The relationship between anxiety and depression. Clinical Psychology Review, 5, 307-324.

Green, D. P., Goldman, S. L., y Salovey, P. (1993). Measurement error masks bipolarity in affect ratings. Journal of Personality and Social Psychology, 64, 1029-1041.

Jackson, S. W. (1985). Acedia the sin and its relationship to sorrow and melancholia. En A. Kleinman y B. Good (Eds.), Culture and depression (pp. 43-62). Berkeley, CA: University of California Press.

Joiner, Jr., T. E. (in press). A confirmatory factor analytic investigaton of the tripartite model of depression and anxiety among college undergraduates. Cognitive Therapy and Research.

Joiner, Jr., T. E., y Blalock, J. A. (in press). Gender differences in depression: The role of anxiety and generalized negative affect. Sex Roles.

Joiner, Jr., T. E., Blalock, J. A., y Wagner, K. D. (1995). Gender differences in depression among adolescent psychiatric inpatients: The roles of negative affect and anxiety. Manuscript under editorial review.

Joiner, Jr., T. E., Catanzaro, S., y Laurent, J. L. (En prensa). The tripartite structure of positive and negative affect, depression, and anxiety in child and adolescent psychiatric inpatients. Journal of Abnormal Psychology

Joiner, Jr., T. E., Sandin, B., Chorot, P., Lostao, L., y Marquina, G. (1995). Development and preliminary validation of a Spanish-language measure of mood:(More) cross-cultural convergence in the structure of mood. Manuscipt under editorial review.

Jolly, J. B., y Dykman, R. A., (1994). Using self-report data to differentiate anxious and depressive symptoms in adolescents: Cognitive content specificity and global distress? Cognitive Therapy and Research, 18, 25-38.

Kendall, P. C., Kortlander, E., Chansky, T. E., y Brady, E. U. (1992). Comorbidity of anxiety and 
depression in youth: Treatment implications. Journal of Consulting and Clinical Psychology, $60,869-880$.

Klerman, G. L., y Weissman, M. M. (1989). Increasing rates of depression. Journal of the American Medical Association, 261, 2229-2235.

Leon, G., Kanfer, R., Hoffiman, R. G., y Dupre, L. (1991) Interrelationships of personality and coping in a challenging extreme situation. Journal of Research in Personality, 25, 357-371.

McGrath, E., Keita, G. P., Strickland, B. R., y Russo, N. F. (Eds., 1991). Women and depression: Risk factors and treatment issues. Washington, DC: American Psychological Association.

Nolen-Hoeksema, S., Morrow, J., y Fredrickson, B. L. (1993). Response styles and the duration of episodes of depressed mood. Journal of Abnormal Psychology, 102, 20-28.

Ochoa, L., Beck, A. T., y Steer, R. A. (1992). Gender differences in comorbid anxiety and mood disorders. American Journal of Psychiatry, 149, 1409-1410.

Russell, J. A. (1983). Pancultural aspects of human conceptual organization of emotions. Journal of Personality and Social Psychology, 45, 12811288.

Sánchez-Cánovas, J. (1994). Relaciones entre salud física, psíquica y estrés, estilos de vida saludables y apoyo social: Un estudio exploratorio en mujeres climatéricas. Manuscrito no publicado.

Sánchez-Cánovas, J. y Sánchez, M. P. (1994a). Psicologia diferencial: Diversidad $\theta$ individualidad humanas. Prácticas. Madrid: Editorial Centro de Estudios Ramón Areces.

Sánchez-Cánovas, J. y Sánchez, M. P. (1994b). Psicologia diferencial: Diversidad e individualidad humanas. Madrid: Editorial Centro de Estudios Ramón Areces.
Stark, K. D., Rouse, L. W., y Livingston, R. (1991). Treatment of depression during childhood and adolescence: Cognitive-behavioral procedures for the individual and family. In P. C. Kendall (Ed.), Child and adolescent therapy: Cognitivebehavioral approaches (pp. 165-198). New York: Guilford Press.

Watson, D. (1988). The vicissitudes of mood measurement: Effects of varying descriptors, time frames, and response formats on measures of positive and negative affect. Journal of Personality and Social Psychology, 54, 10201030.

Watson, D., Clark, L. A., y Carey, G. (1988). Positive and Negative Affectivity and their relation to anxiety and depressive disorders. Journal of Abnormal Psychology, 97, 346-353.

Watson, D., Clark, L. A., y Tellegen, A. (1984). Crosscultural convergence in the structure of mood: A Japanese replication and a comparison with U. S. findings. Journal of Personality and Social Psychology, 47, 127-144.

Watson, D., Clark, L. A., y Tellegen, A. (1988). Development and validation of brief measures of Positive and Negative Affect: The PANAS scales. Journal of Personality and Social Psychology, 54, 1063-1070.

Watson, D., Clark, L. A., Weber, K., Assenheimer, J. S., Strauss, M. E., y McCormick, R. A. (1995). Testing a tripartite model: II. Exploring the symptom structure of anxiety and depression in student, adult, and patient samples. Journal of Abnormal Psychology, 104, 15-25.

Zinbarg, R. E., Barlow, D. H., Liebowitz, M., Street, L., Broadhead, E., Katon, W., Roy-Byrne, P., Lepine, J., Teherani, M., Richards, J., Brantley, P. J., y Kraemer, H. (1994). The DSM-IV field trial for mixed anxiety-depression. American Journal of Psychiatry, 151, 1153-1162. 